\title{
Multidisciplinary Management of Radiation-Induced Salivary Gland Carcinomas in the Modern Radiotherapy Era
}

\author{
Domenico Attilio Romanello ${ }^{1,2}$, Zulfiyya Imamguliyeva ${ }^{3}$, Stefano Cavalieri ${ }^{4, *(\mathbb{D} \text {, }}$ \\ Barbara Vischioni ${ }^{1}$, Lorenza Gandola ${ }^{5}$, Alberto Iannalfi ${ }^{1}$, Nicola Alessandro Iacovelli ${ }^{6}$, \\ Lisa Licitra ${ }^{4,7}$, Marco Guzzo $^{8}$, Cesare Piazza ${ }^{9}$, Davide Lombardi ${ }^{9} \mathbb{D}$, Barbara Diletto ${ }^{6}$, \\ Pasquale Quattrone ${ }^{10}$, Giuseppina Calareso ${ }^{11}$, Laura Deborah Locati ${ }^{4,+}$ and Ester Orlandi ${ }^{1,+}$ \\ 1 Radiotherapy Unit, National Center of Oncological Hadrontherapy (CNAO), 27100 Pavia, Italy; \\ domenico.romanello@cnao.it (D.A.R.); barbara.vischioni@cnao.it (B.V.); alberto.iannalfi@cnao.it (A.I.); \\ ester.orlandi@cnao.it (E.O.) \\ 2 School of Medicine, University of Milan-Bicocca, 20126 Milan, Italy \\ 3 Department of Head and Neck Tumors, National Center of Oncology, Baku AZ1012, Azerbaijan; \\ dr.imamguliyeva@gmail.com \\ 4 Head and Neck Cancer Medical Oncology 3 Unit, Fondazione IRCCS Istituto Nazionale dei Tumori \\ di Milano, 20133 Milan, Italy; lisa.licitra@unimi.it (L.L.); laura.locati@istitutotumori.mi.it (L.D.L.) \\ 5 Radiotherapy 1 Unit, Fondazione IRCCS Istituto Nazionale dei Tumori, 20133 Milan, Italy; \\ lorenza.gandola@istitutotumori.mi.it \\ 6 Radiotherapy 1-2 Unit, Fondazione IRCCS Istituto Nazionale dei Tumori, 20133 Milan, Italy; \\ nicolaalessandro.iacovelli@istitutotumori.mi.it (N.A.I.); barbara.diletto@istitutotumori.mi.it (B.D.) \\ 7 Department of Oncology and Hemato-Oncology, University of Milan, 20126 Milan, Italy \\ 8 Department of Otorhinolaryngology, Maxillofacial, and Thyroid Surgery, Fondazione IRCCS Istituto \\ Nazionale dei Tumori, 20133 Milan, Italy; marco.guzzo@istitutotumori.mi.it \\ 9 Unit of Otorhinolaryngology-Head and Neck Surgery, Department of Medical and Surgical Specialties, \\ Radiological Sciences, and Public Health, University of Brescia, 25123 Brescia, Italy; \\ cesare.piazza@unibs.it (C.P.); davinter@libero.it (D.L.) \\ 10 Pathology Department, Fondazione IRCCS Istituto Nazionale dei Tumori di Milano, 20133 Milan, Italy; \\ pasquale.quattrone@istitutotumori.mi.it \\ 11 Department of Radiology, Fondazione IRCCS Istituto Nazionale dei Tumori di Milano, 20133 Milan, Italy; \\ giuseppina.calareso@istitutotumori.mi.it \\ * Correspondence: stefano.cavalieri@istitutotumori.mi.it \\ $\dagger$ These authors contributed equally to this paper as senior co-last authors.
}

Received: 25 November 2020; Accepted: 10 December 2020; Published: 14 December 2020

check for updates

Simple Summary: Etiopathogenesis of salivary gland cancers [SGCs] is largely unknown, even if exposition to ionizing radiation is a recognized risk factor for SGCs development. To date, exhaustive data to guide clinicians in managing patients with radiation-induced [ri] SGCs are scarce and their treatment remains challenging. The purpose of this work is to describe and to analyze clinical and histopathological features, delivered treatments, and outcome of a series of patients with ri-SGCs treated at two Italian cancer referral sites. Given the rarity of ri-SGCs, this retrospective analysis conducted on a case series of 13 patients adds further knowledge to the paucity of literature. The management of these malignancies is extremely complex requiring a multidisciplinary treatment approach.

Abstract: Clinical data of ri-SGCs patients treated between 2015 and 2019 at a tertiary cancer center and a national hadron therapy facility were reviewed. Latent time (LT) from first RT to ri-SGCs diagnosis, overall (OS), and disease-free survival (DFS) were assessed. Thirteen patients developed 14 ri-SGCs (one patient had 2 synchronous ri-SCGs), after a median LT of 23 years (range 16-34). Parotid was the primary site in 8 cases $(57 \%)$ and salivary duct carcinoma was the most frequent 
histotype (29\%). Nine patients (69\%) underwent surgery (Sx). Among them, 4 patients $(31 \%)$ underwent Sx alone, 5 received post-operative treatments: $3(23 \%)$ photon-based $(X)$ reRT, one $(8 \%)$ protons and carbon ions, one ( $8 \%$ ) carbon ions only. One patient $(8 \%)$ received definitive XRT. The remaining 3 patients $(23 \%)$ received androgen deprivation therapy. With a median follow-up of 48 months (range 24-72), median OS and PFS were 74 and 24 months, respectively. In the subgroup of $\mathrm{AR}^{+}$ri-SGCs, median PFS and OS were 12 and 74 months, respectively. Given the rarity of ri-SGCs, this work adds further knowledge to the paucity of literature. The management of these malignancies is extremely complex requiring a multidisciplinary treatment approach.

Keywords: Radiation induced cancer; salivary gland cancer; multidisciplinary management; radiotherapy; particle therapy; hormone therapy; surgery

\section{Introduction}

Malignant tumors of salivary glands (SGCs) comprise less than $0.5 \%$ of all cancers and constitute about $2-8.5 \%$ of head and neck cancers (HNCs) [1]. Worldwide annual incidence ranges from 0.4 to 2.6 cases over 100,000 [2] with a male to female ratio of 1.5:1, a higher prevalence in males compared to females during the 6th-7th decade for the salivary duct carcinoma (SDC), being exceedingly uncommon in children [1-3].

Etiopathogenesis of SGCs is largely unknown, even if exposition to ionizing radiation is a recognized risk factor for SGCs development. This was reported for the first time in Japanese survivors of the atomic bomb: the frequency of SGCs, mucoepidermoid carinoma (MEC) in particular, was disproportionately high at high radiation doses. In Japanese survivors, out of 145 SGCs registered from 1950 to 1987, 41 were malignant and the proportion of MEC raised with the increasing of radiation dose $(p=0.004$ for linear trend) [4]. However, even doses of 2 Gy could raise the risk of developing salivary gland tumors $[4,5]$.

Further evidence supporting the role of radiation in salivary gland carcinogenesis derives from data of children treated with scalp irradiation for tinea capitis, or who received radiotherapy (RT) to the head and neck area to reduce the size of the tonsils and adenoids [6,7]. These children had a 4.5-fold incidence of SGCs compared to untreated people with a mean latency period until tumor development of 11 years [6].

Globally, the standardized incidence ratio of HNCs in survivors of childhood cancer is 13.6 and SGCs represent $65 \%$ of these cases [8]. The risk has been associated with the primary tumor type, gender, type of chemotherapy, etc. Radiation has been associated with an increased risk of all carcinomas, most marked for HNCs (SIR 18.5), and the site of the second tumor had arisen in a previous radiation field in $85 \%$ of HNCs. Besides, a large study on children survivors after RT (with or without chemotherapy) for a first cancer, showed a linear correlation between dose exposure and the risk of developing solid tumors including SGCs, with the second malignancy occurring at least eight years after the end of the first oncological treatment [9].

Exhaustive data to guide clinicians in managing patients with ri-SGCs are scarce and their treatment remains challenging, especially in the era of targeted systemic treatments and innovative radiation therapies, namely intensity modulated RT (IMRT) and hadron therapy [9-13].

Ideally, surgery with wide margins remains the mainstay of treatment followed by RT in high-risk cases $[14,15]$. However, radical salvage surgery is often not feasible, essentially because of technical difficulties when operating within an irradiated area $[9,12,16]$ or due to the local extension of disease that prevents a radical resection.

In addition, further RT could be hardly given since normal tissues surrounding secondary SGCs have been generally treated to near their dose tolerance. Finally, in some cases, patients who suffer from secondary SGCs are young and potentially candidates to develop a third cancer, in particular in the presence of a genetic susceptibility [17]. In this scenario, modern RT techniques, including IMRT and particle therapy, may play a principal role in the management of these patients. Indeed, thanks to their physical properties, they have an excellent sparing of normal tissue outside the target and they are able to overcome an enhanced hypo-oxygenation that can also be present in ri-SGCs [14]. 
With the limitations of the descriptive nature of our work and the limited patient cohort, the purpose of our review is to describe and to analyze clinical and histopathological features, delivered treatments, and outcome of a series of patients with ri-SGCs.

\section{Results}

\subsection{Patients' Characteristics}

Patients' clinical characteristics at the first cancer diagnosis are listed in Table 1.

Overall, we have identified 13 patients suitable for this analysis. One of these patients had two synchronous secondary SGCs after a LT of 34 years, for a total of 14 ri-SGC cases. Median age at diagnosis of primary tumor was 17 years (range 3-68), whereas median age at diagnosis of ri-SGC was 50 years (range 17-78), with a median interval between radiation exposure and diagnosis of the SGCs of 23 years (95\% CI: 16-34).

Characteristics of the second SGCs were resumed in Table 2. Histology was quite heterogeneous: low grade MEC $(n=2)$, SDC $(n=4)$, low-grade polymorphous adenocarcinoma $(n=1)$, adenocarcinoma not otherwise specified NOS) $(n=3)$, carcinoma ex- pleomorphic adenoma $(n=1)$, adenoid-cystic carcinoma (ACC) $(n=2)$, basal cell adenocarcinoma $(n=1)$. Eight tumors occurred in the parotid glands, five in submandibular glands, one in minor salivary glands of the oropharynx. Positive nodes $(n=3)$ have been detected in submandibular $(n=2)$ or minor salivary glands $(n=1)$ tumors whereas distant metastases were present in 2 cases. Median LT to ri-SGCs was not significantly different whether primary tumor was diagnosed in pediatric age (31 years, range 5-50) or in adult (33.5 years, range 18-68), even though in the former diagnosis of ri-SGCS appeared earlier after primary treatment compared to the latter.

Immunohistochemical (IHC) research of androgen receptor has been done only in cases with diagnosis of SDC, adenocarcinoma NOS, and carcinoma ex pleomorphic adenoma. Seven out of 8 patients had androgen receptors (ARs) overexpression. HER2 overexpression was not identified. NGS was performed in 6 out of $13(46 \%)$ cases, in 3 cases somatic tumor mutations have been found: BRCA2 mutation in one SDC, TP53 mutation in one adenocarcinoma NOS, PIK3CA and HRAS mutations in another SDC.

Concerning the relationship with the previous treated volumes, data were available for $9(64 \%)$ ri-SGCs (one patient had two synchronous ri-SGCs, (Table 1). Six ri-SGCs developed within the previously treated volumes and in 5 of them, ri-SGC was marginal to high-dose volumes. Three ri-SGCs occurred within the initial target volume.

\subsection{Treatment and Outcome for Secondary SGCs}

Nine patients $(69 \%)$ underwent surgery, either alone $(n=3)$, in combination with RT $(n=5)$, or with postoperative systemic treatment $(n=1)$; resection margins were positive in 8 cases. One patient received definitive RT. The remaining 3 patients received palliative treatment.

Seven patients $(54 \%)$ out of 13 received RT as part of their multimodality treatment. Post-operative photon-based RT (XRT) $(n=3)$ doses ranged from 64 Gy to 70 Gy (median of 67 Gy); one patient received a palliative course of $30 \mathrm{~Gy}$ for an unresectable parotid gland tumor. Particle therapy was delivered in two cases: one patient was treated by post-operative Proton Therapy (PT) with a boost of Carbon Ion Radiotherapy (CIRT), receiving 6 Gy(RBE) with CIRT and 59.4 Gy with PT. The other one was treated with post-operative CIRT after microscopic residual surgery, with a total dose of $68.8 \mathrm{~Gy}(\mathrm{RBE})$. In the subgroup treated with post-operative RT, median age at primary tumor diagnosis was 12 years (range 3-27) and median LT was 17 years (range 5-41 years). The patient treated with radical RT was 8 years old at the time of the diagnosis of the primary tumor, with an LT of 37 years. With regard to acute toxicities, all of the patients who underwent post-operative RT developed mild to moderate xerostomia and oral mucositis $(\leq \mathrm{G} 2)$; as regards late toxicities, three patients treated with surgery and XRT developed buccal spasms $(n=1)$, fibrosis $(n=1)$, trismus and neuropathy of the facial nerve $(n=2)$. Both the 2 patients treated with post-operative particle therapy developed G1 trismus, G1 xerostomia, and G1 neuropathy of the facial and trigeminal nerve. 
Table 1. Patient characteristics at diagnosis of primary cancer.

\begin{tabular}{|c|c|c|c|c|c|c|c|c|c|}
\hline Patient & Gender & $\begin{array}{c}\text { Age at } \\
\text { Primary Diagnosis }\end{array}$ & $\begin{array}{c}\text { Year of } \\
\text { First Diagnosis } \\
\end{array}$ & Histology & Site and Stage & Treatment & RT Techniques/RT Volumes and Doses & Late Sequelae & $\begin{array}{l}\text { Latent Time between } \\
\text { Primary Tumor and SGCs }\end{array}$ \\
\hline Patient 1 & $\mathrm{~F}$ & 10 & 2000 & Medulloblastoma & $\begin{array}{l}\text { Cerebellar vermis T3M0 } \\
\text { (Chang system) } \\
\text { High Risk ( }\left(>1.5 \mathrm{~cm}^{2}\right. \\
\text { residual disease) }\end{array}$ & $\begin{array}{c}\text { Sequential high dose } \\
C T \rightarrow S x \rightarrow>R T \\
\text { Vincristine and CCNU-based } \\
\text { maintenance therapy }\end{array}$ & $\begin{array}{c}\text { Conventional XRT } \\
\text { Hyperfractionated accelerated RT (HART) } \\
\text { Craniospinal axis: } 39 \mathrm{~Gy} \\
\text { Posterior fossa: } 60 \mathrm{~Gy}\end{array}$ & $\begin{array}{l}\text { Neurocognitive impairment } \\
\text { Panhypopituitarism } \\
\text { Bilateral hypoacusia }\end{array}$ & 16 years \\
\hline Patient 2 & M & 68 & 2008 & $\begin{array}{l}\text { Undifferentiated carcinoma } \\
\text { EBER-negative HPV-positive }\end{array}$ & $\begin{array}{c}\text { Nasopharynx } \\
\text { cT2N2c (AJCC VII ed) }\end{array}$ & Platinum-based CRT & $\begin{array}{c}\text { IMRT } \\
\text { Elective nodal volume: } 50 \mathrm{~Gy} \\
\text { High risk volume: } 70 \mathrm{~Gy} \\
\text { Conventional fractionation }\end{array}$ & $\begin{array}{l}\text { Severe Xerostomia } \\
\text { Dental alterations } \\
\text { Moderate fibrosis }\end{array}$ & 10 years \\
\hline Patient 3 & M & 18 & 1985 & $\begin{array}{l}\text { Nodular } \\
\text { lymphocyte-predominant } \\
\text { HL }\end{array}$ & $\begin{array}{c}\text { Neck } \\
\text { Stage IIA } \\
\text { (Ann Arbor staging) }\end{array}$ & Neck Sx $\rightarrow$ RT & $\begin{array}{l}\text { Conventional XRT: } \\
40 \text { Gy mantle field } \\
45 \text { Gy neck nodes }\end{array}$ & $\begin{array}{l}\text { Chronic ischemic } \\
\text { cardiomyopathy }\end{array}$ & 32 years \\
\hline Patient 4 & M & 40 & 1992 & Squamous cell carcinoma & Oropharynx & Tonsillectomy $\rightarrow$ RT & $\begin{array}{l}\text { Conventional XRT } \\
\text { Elective nodal volume: } 45 \mathrm{~Gy} \\
\text { High risk volume: } 60 \mathrm{~Gy} \\
\text { Conventional fractionation }\end{array}$ & $\begin{array}{l}\text { Xerostomia } \\
\text { Hypothyroidism }\end{array}$ & 23 years \\
\hline Patient 5 & $\mathrm{~F}$ & 27 & 1975 & Papillary thyroid carcinoma & $\begin{array}{c}\text { Thyroid } \\
\text { (stage not available) } \\
\end{array}$ & $\begin{array}{c}\text { Total thyroidectomy > } \\
\text { external beam RT }\end{array}$ & $\begin{array}{l}\text { Conventional } \gamma \text { photon-based RT (Co60) } \\
45 \text { Gy }\end{array}$ & $\begin{array}{c}\text { Xerostomia } \\
\text { Moderate fibrosis }\end{array}$ & 41 years \\
\hline Patient 6 & M & 10 & 1997 & Sarcoma G3, NOS & $\begin{array}{c}\text { Soft tissues of } \\
\text { paramandibular area } \\
\text { (locally advanced disease, } \\
\text { stage not available) }\end{array}$ & $\begin{array}{c}\mathrm{Sx} \rightarrow \mathrm{CT}(\mathrm{CEVAIE}) \rightarrow \mathrm{RT} \\
(44.8 \mathrm{~Gy}, 1.6 \text { Gy twice a day })\end{array}$ & Conventional XRT & Moderate fibrosis & 19 years \\
\hline Patient 7 & M & 8 & 1975 & $\mathrm{HL}$ & $\begin{array}{c}\text { Neck Stage II } \\
\text { (Ann Arbor staging) }\end{array}$ & $\mathrm{CT} \rightarrow \mathrm{RT}$ & Conventional XRT & Moderate fibrosis & 37 years \\
\hline Patient 8 & $\mathrm{~F}$ & 17 & 1985 & $\begin{array}{l}\text { Undifferentiated } \\
\text { Carcinoma } \\
\text { (EBER unknown) }\end{array}$ & $\begin{array}{c}\text { Nasopharynx } \\
\text { (loco-regionally advanced } \\
\text { disease, stage not available) }\end{array}$ & $\begin{array}{c}\text { CT (Vincristine, } \\
\text { Adriamycin and } \\
\text { cyclophosphamide } \times 2 \text { cycles) } \\
\text { RT } \\
+ \text { concurrent adriamycin and } \\
\text { cyclophosphamide } \times 4 \text { cycles }\end{array}$ & $\begin{array}{l}\text { Conventional XRT } \\
\text { T and pathological nodes: } 62 \mathrm{~Gy} \\
\text { Elective nodes: } 40.2 \mathrm{~Gy}\end{array}$ & $\begin{array}{c}\text { Trisma } \\
\text { Xerostomia } \\
\text { Massive fibrosis } \\
\text { ipoplasia mandibola }\end{array}$ & 34 years \\
\hline Patient 9 & м & 19 & 1993 & $\begin{array}{l}\text { Undifferentiated carcinoma } \\
\text { (EBER unknown) }\end{array}$ & $\begin{array}{c}\text { Nasopharynx } \\
\text { T4N1 } \\
\text { (AJCC VII edition) } \\
\end{array}$ & Exclusive RT & Conventional XRT & $\begin{array}{c}\text { Neck fibrosis } \\
\text { Facial asymmetry } \\
\text { Sinonasal synechiae } \\
\end{array}$ & 22 years \\
\hline Patient 10 & $\mathrm{~F}$ & 12 & 2014 & $\mathrm{HL}$ & $\begin{array}{c}\text { Neck } \\
\text { Stage III } \\
\text { (Ann Arbor staging) }\end{array}$ & $\begin{array}{l}\text { CT (COPP/ABV } \times 6) ; \\
\text { RT above and } \\
\text { under diaphragm } \\
\text { (total dose } 14.4 \mathrm{~Gy})\end{array}$ & $\begin{array}{l}\text { Conventional XRT } \\
\text { T and pathological nodes: } 62 \mathrm{~Gy}, \\
\text { elective nodes: } 40.2 \mathrm{~Gy}\end{array}$ & - & 5 years \\
\hline Patient 11 & M & 3 & 1983 & $\mathrm{HL}$ & $\begin{array}{c}\text { Neck } \\
\text { Stage IV (Ann Arbor } \\
\text { staging) for bone } \\
\text { marrow infiltration }\end{array}$ & $\mathrm{CT}+\mathrm{RT}$ & $\begin{array}{l}\text { Conventional XRT } \\
20 \mathrm{~Gy}\end{array}$ & - & 31 years \\
\hline Patient 12 & M & 45 & 1993 & $\begin{array}{l}\text { Undifferentiated carcinoma } \\
\text { (EBER unknown) }\end{array}$ & $\begin{array}{l}\text { Nasopharynx } \\
\text { (stage unknown) }\end{array}$ & $\begin{array}{l}\text { Induction cisplatin }+5-\mathrm{FU} \\
\text { followed by exclusive } \mathrm{RT}\end{array}$ & Conventional XRT & - & 19 years \\
\hline Patient 13 & M & 13 & 1963 & Lymphoma NOS & $\begin{array}{l}\text { Supradiaphragmatic } \\
\text { lymph nodes }\end{array}$ & $\mathrm{RT}$ above diaphragm & $\begin{array}{c}\text { Conventional XRT } \\
\text { mantle field } \\
\text { (unknown total dose) }\end{array}$ & - & 50 years \\
\hline
\end{tabular}

Legend: 5-FU, 5-fluorouracil; ABV, adriamycin, bleomycin, vinblastine; AJCC, American Joint Committee on Cancer; CEVAIE, carboplatin, epirubicin, vincristine, actinomycin, ifosfamide, etoposide; COPP, cyclophosphamdie, vincristine, procarbazine, prednisone; CRT, concomitant chemoradiation; CT, chemotherapy; EBER, Epstein-Barr virus-encoded RNA;

HL, Hodgkin lymphoma; NOS, not otherwise specified; RT, external beam radiotherapy; Sx, surgery; XRT, photon-based radiotherapy. 
Table 2. Patient characteristics at clinical presentation of radiation-induced SGC and at follow-up.

\begin{tabular}{|c|c|c|c|c|c|c|c|c|c|c|c|c|}
\hline Patient & $\begin{array}{c}\text { Age at } \\
\text { Secondary Diagnosis }\end{array}$ & $\begin{array}{c}\text { Year of } \\
\text { SGC Diagnosis }\end{array}$ & Site and Stage & SGCs Histology & Molecular Analysis & $\begin{array}{c}\text { Relationship between SGC } \\
\text { Volume and Previous RT } \\
\text { Doses and Target Volumes } \\
\end{array}$ & Treatment of SGCs & $\begin{array}{l}\text { Second Course of RT } \\
\text { Techniques/RT Dose } \\
\text { and Volume }\end{array}$ & Recurrence//Progression & Toxicity & Last Follow-Up Status & os Time \\
\hline Patient 1 & 29 & 2016 & $\begin{array}{l}\text { Parotid gland } \\
\text { PT1 }\end{array}$ & $\begin{array}{l}\text { Low Grade Polymorphous } \\
\text { Adenocarcinoma }\end{array}$ & N.A. & $\begin{array}{c}\text { Within initial treatment } \\
\text { volume and marginal to } \\
\text { high-dose volume }\end{array}$ & $\mathrm{Sx}(\mathrm{R} 1) \rightarrow \mathrm{RT}$ & $\begin{array}{l}\text { Photon-based VMAT } \\
64 \text { Gy o pr partidi } \\
\text { surgical bed }\end{array}$ & No & Buccal spasms & $\begin{array}{l}\text { Alive } \\
\text { NED }\end{array}$ & 36 months \\
\hline Patient 2 & 78 & 2018 & $\begin{array}{c}\text { Parotid gland } \\
\text { cT4 } \\
\text { Unresectable disease }\end{array}$ & $\begin{array}{l}\text { Salivary duct carcinoma } \\
\mathrm{AR}^{2}+\end{array}$ & $\begin{array}{l}\text { PIK3CA: p.E542K exon } 9 \\
\text { (6\% of DNA) } \\
\text { HRAS: p. P61R exon } 3 \\
\text { (13\% of ofNA) } \\
\text { HER-2 negative }\end{array}$ & $\begin{array}{l}\text { Within elective volume and } \\
\text { marginal high dose volume }\end{array}$ & $\mathrm{ADT} \rightarrow$ palliative RT & $\begin{array}{l}\text { Photon based VMAT } \\
\text { 30 Gy to macroscopic parotid } \\
\text { disease (3 Gy per fraction) }\end{array}$ & $\begin{array}{l}\text { Local progression after } \\
2 \text { months }\end{array}$ & - & $\begin{array}{c}\text { Dead } \\
\text { ED }\end{array}$ & 13 months \\
\hline Patient 3 & 50 & 2017 & $\begin{array}{l}\text { Right parotid gland } \\
\text { CT4 } \\
\text { Unresectable disease }\end{array}$ & $\begin{array}{l}\text { Salivary duct carcinoma } \\
\mathrm{AR+}\end{array}$ & HER-2 negative & $\begin{array}{l}\text { Within initial treatment } \\
\text { volume }\end{array}$ & $\mathrm{ADT}$ & Not delivered & $\begin{array}{l}\text { Local progression and } \\
\text { bone metastasis after } \\
6 \text { months }\end{array}$ & - & $\begin{array}{c}\text { Alive } \\
\mathrm{ED}\end{array}$ & 24 months \\
\hline Patient 4 & 63 & 2015 & $\begin{array}{l}\text { Right submandibular gland. } \\
\text { Stage pT1N0 }\end{array}$ & $\begin{array}{l}\text { Salivary duct carcinoma } \\
\mathrm{AR+}\end{array}$ & HER-2 negative & Not applicable & $\begin{array}{l}\text { Surgery for local disease. } \\
\text { At diagnosis of } \\
\text { metastatic disease } \\
\text { chemotherapy } \\
\text { (CBDCA/paclitaxel), } \\
\text { followed by ADT }\end{array}$ & Not delivered & 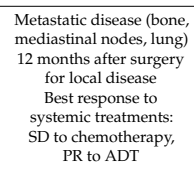 & - & $\begin{array}{l}\text { Alive } \\
\mathrm{ED}\end{array}$ & 48 months \\
\hline Patient 5 & 68 & 2016 & $\begin{array}{c}\text { Parotid } \\
\text { pT3R2 } \\
\text { (RP nodes } \\
\text { radiologically positive) }\end{array}$ & $\begin{array}{c}\text { Carcinoma ex } \\
\text { pleomorphica adenoma } \\
\text { AR- }\end{array}$ & NR4A3 negative & Marginal to target volume & $S_{x} \rightarrow R T$ & $\begin{array}{l}\text { Photon based VMAT-SIB in } \\
33 \text { fractions } \\
69.96 \mathrm{G} \text { on } \\
\text { macroscopic disease+ } \\
59.4 \text { Gy RP nodes and I-II } \\
\text { neck levels }\end{array}$ & No & $\begin{array}{c}\text { Fibrosis } \\
\text { Trismus } \\
\text { Facial nerve disorder }\end{array}$ & $\begin{array}{l}\text { Alive } \\
\text { NED }\end{array}$ & 38 months \\
\hline Patient 6 & 27 & 2015 & $\begin{array}{c}\text { Left parotid } \\
\text { PT3 R2 }\end{array}$ & $\begin{array}{l}\text { Low grade } \\
\text { mucoepidermoid carcinoma }\end{array}$ & N.A. & Marginal to target volume & $S x \rightarrow R T$ & $\begin{array}{c}\text { Photon based VMAT-SEQ in } \\
3 \text { fractions } \\
70 \text { Gy or residal disease } \\
50 \text { Gy on surgical bed }\end{array}$ & No & $\begin{array}{l}\text { Trismus } \\
\text { Facial nerve disorder }\end{array}$ & $\begin{array}{l}\text { Alive } \\
\text { NED }\end{array}$ & 48 months \\
\hline Patient 7 & 42 & 2012 & $\begin{array}{c}\text { Submandibular cT4 } \\
\text { (ipsilateral mandibular } \\
\text { nerve involvement) } \\
\text { Unresectable disease }\end{array}$ & $\begin{array}{l}\text { Adenocarcinoma NOS } \\
\mathrm{AR+}^{+}\end{array}$ & N.A. & Marginal to target volume & $\mathrm{RT}+\mathrm{ADT}$ & $\begin{array}{l}\text { Photon based VMAT-SEQ in } \\
30 \text { fractions }\end{array}$ & $\begin{array}{l}\text { Brain and bone } \\
\text { Progression after } \\
30 \text { months }\end{array}$ & - & $\begin{array}{c}\text { Dead } \\
\text { ED }\end{array}$ & 36 months \\
\hline Patient 8 & 50 & 2019 & $\begin{array}{l}\text { Left submandibular gland } \\
\text { pT4R2pN1ECS } \\
\text { Right submandibular gland } \\
\text { PT4R2pNo }\end{array}$ & $\begin{array}{l}\text { Adenoid cystic carcinoma } \\
\text { (solid variant) } \\
\text { Basal cell adenocarcinoma }\end{array}$ & MYB negative (ACC) & $\begin{array}{c}\text { Marginal to high-risk } \\
\text { target onumes } \\
\text { within elective volume } \\
\text { Marginat th high-irisk } \\
\text { target volume }\end{array}$ & $5 x$ & Not delivered & $\begin{array}{c}\text { Stable disease at } \\
\text { both sides }\end{array}$ & - & $\begin{array}{c}\text { Alive } \\
\mathrm{ED}\end{array}$ & 4 months \\
\hline Patient 9 & 41 & 2016 & $\begin{array}{c}\text { Minor salivary } \\
\text { gland (Oropharynx) } \\
\text { PT2R1N2bECS } \mathrm{M1} \text { (bone) }\end{array}$ & $\begin{array}{l}\text { Adenocarcinoma NOS } \\
\mathrm{AR+}+\end{array}$ & $\begin{array}{c}\text { TP53: p.V216M exon } 6 \\
\text { (13\% of DNA } \\
\text { HER-2 negative }\end{array}$ & Not applicable & $\mathrm{Sx} \rightarrow \mathrm{ADT}$ & Not delivered & $\begin{array}{c}\text { Bone lung brain } \\
\text { progression after } \\
25 \text { months from surgery }\end{array}$ & & $\begin{array}{c}\text { Dead } \\
\mathrm{ED}\end{array}$ & 39 months \\
\hline Patient 10 & 17 & 2019 & $\begin{array}{l}\text { Parotid } \\
\text { PT2 R2 }\end{array}$ & $\begin{array}{l}\text { Low Grade } \\
\text { Mucoepidermoid carcinoma }\end{array}$ & N.A. & $\begin{array}{l}\text { Marginal to high--risk } \\
\text { withre et volume } \\
\text { withelective volume }\end{array}$ & $\mathrm{Sx} \rightarrow \mathrm{RT}$ & $\begin{array}{l}\text { CIRT } 6 \text { Gy }>\text { Proton therapy } \\
559.4 \text { Gy to parotid bed, } \\
50.4 \text { Gy to perineural path }\end{array}$ & No & $\begin{array}{c}\text { Trismus } \\
\text { Xerstomia } \\
\text { Facial and trigeminal } \\
\text { nerve disorder }\end{array}$ & $\begin{array}{l}\text { Alive } \\
\text { NED }\end{array}$ & 11 months \\
\hline Patient 11 & $34(23$ thyroid cancer) & $\begin{array}{l}\text { December, } 2013 \\
\text { 2005: } \\
\text { thiroidectomy } \\
\text { and RAI for } \\
\text { papillary ca } \\
\end{array}$ & $\begin{array}{l}\text { Parotid } \\
\text { pT3R1 }\end{array}$ & $\mathrm{ACC}$ & $\begin{array}{l}\text { NGS: no mutations } \\
\text { ALK and ROS1 negative }\end{array}$ & Not applicable & $\mathrm{Sx} x \rightarrow \mathrm{RT}$ & $\begin{array}{c}\text { CIRT 6.8.8 Gy(RBE) } \\
(4.3 \text { Gy (RBE) } 16 \text { over } \\
\text { 4 weeks) }\end{array}$ & $\begin{array}{l}\text { Lung metastases } 2 \text { years } \\
\text { after surgery for ACC } \\
\text { Currently active } \\
\text { surveilince of fung } \\
\text { metastases }\end{array}$ & $\begin{array}{l}\text { Trismus Xerostomia } \\
\text { Facial and trigeminal } \\
\text { nerve disorder }\end{array}$ & $\begin{array}{c}\text { Alive } \\
\mathrm{ED}\end{array}$ & 72 months \\
\hline Patient 12 & 64 & 2012 & Left parotid M1 (lung) & $\begin{array}{c}\text { Salivary duct carcinoma } \\
\text { AR+ }\end{array}$ & $\begin{array}{l}\text { NCS: BRCA2: P.A336T } \\
\text { exon } 10 \text { (50\% of DNA) }\end{array}$ & Not applicable & Chemotherapy & Not delivered & $\begin{array}{c}\text { Bone, lung and brain } \\
\text { progression }\end{array}$ & - & $\begin{array}{c}\text { Alive } \\
\text { ED }\end{array}$ & 65 months \\
\hline Patient 13 & 63 & 2013 & $\begin{array}{l}\text { Right submandibular gland } \\
\text { pT4a pN2b }\end{array}$ & $\begin{array}{c}\text { Adenocarcinoma NOS } \\
\text { AR+ }\end{array}$ & NGS: no mutations & Not applicable & $5 x$ & Not delivered & $\begin{array}{l}\text { Loco-regional, lung and } \\
\text { pleural progression }\end{array}$ & - & $\begin{array}{c}\text { Dead } \\
\text { ED }\end{array}$ & 74 months \\
\hline
\end{tabular}

Legend: ACC, adenoid cystic carcinoma; ADT, androgen deprivation therapy [bicalutamide + LHRH analogue]; AR, Androgen receptor; AR+, Androgen receptor overexpression; CBDCA, carboplatin; CIRT, carbon ion radiation therapy; ED, evidence of disease; LHRH, luteinizing hormone releasing hormone; N.A., not assessed; NED, no evidence of disease; NGS, next generation sequencing; PR, partial response; RAI, radioactive iodine; RT, radiation therapy; RP, retropharyngeal node; S, surgery; SD, stable disease; SGC, salivary gland cancer; R1, microscopic residual tumor after surgery; R2, macroscopic residual tumor after surgery; ECS+, extracapsular spread. 
Cut-off date was October 2019.

Median follow-up was 48 months (95\% CI: 24-72). Median OS was 74 months (95 \% CI: 36.00-74.00). Median PFS was 24 months (95\%CI: 6.00-upper limit not reached). Five-year OS and DFS were 67\% and $26 \%$, respectively. For the subgroup of $\mathrm{AR}^{+}$SGCs, median DFS and OS were 12 months $(95 \% \mathrm{CI}$ : 2.00-25.00) and 74 months (95\% CI: 13.00-74.00), respectively. In AR ${ }^{-}$SGC patients, median DFS and OS were not reached; 2-year DFS was $62.5 \%$ and 2-year OS $100 \%$.

\section{Discussion}

We reported a small series of 13 patients with ri-SGCs with a more favorable outcome than previously published older series. Median PFS and OS were 24 months (95\% CI: 6 months-not reached) and 74 months (95\% CI: 36-74), respectively, despite high-grade histology in 77\% of cases and advanced stage (III-IV) in 10 out 13 cases. These results are very encouraging, if we consider that 9 patients $(69 \%)$ received surgery, 1 patient $(8 \%)$ was treated with definitive XRT and concomitant androgen deprivation therapy (ADT), 1 patient $(8 \%)$ received palliative XRT and subsequent ADT, whereas the remaining 2 patients (15\%) received only systemic therapy. In the past, a 5-year OS higher than $90 \%$ was reported, however, mucoepidermoid was the most common histotype and almost all patients received surgery combined or not with RT $[9,12]$. In the analysis by Bhattacharyya et al., for example, patients with MECs exhibited a higher 5-year OS (81.5\%), compared to other histotypes such as ACC $(70.7 \%)$ and carcinoma ex pleomorphic adenoma (40.2\%) [18]. On the other hand, Mallik et al. reported 47 submandibular gland cancers, mostly ACCs, with a 5-year DFS of $71.8 \%, 12.8 \%(n=6)$ of loco-regional failure, and $12.8 \%(n=6)$ of development of distant metastases. Compared to the past, availability of new effective systemic treatments (e.g., androgen deprivation therapy, ADT; anti-HER2 monoclonal antibody), improvement in the re-irradiation (reRT) [19] techniques and the opening of heavy ions facility in Italy in 2011 have offered new therapeutic opportunities.

If MEC was the most common histotype [3-7,9,11-13,16,20-25] in previous series, the SGC histotypes reported in our series are quite heterogeneous: 9 out $13(69 \%)$ were high-grade with AR expression in 7 out of 9 cases. Of note, $38 \%$ received ADT with a median PFS and OS of 12 and 74 months, respectively. Activity of androgen blockade in AR expressing SGCs is already well known. Several papers [26-28] support the use of ADT in clinical practice and one randomized, prospective trial, is currently ongoing (NCT01969578). New potential druggable targets as HER2 [29], TRK [30], RET [31], BRAF [32] have been identified almost exclusively in high-risk histologies different from ACC, paving the way to active treatment approaches. Impressive results have been reported in the clinical practice with some tailored agents as trastuzumab emtansine [33], NTRK inhibitors [34,35], or RET inhibitors [36]. Activity of some antiangiogenic agents (e.g., lenvatinib) seems to be promising in ACC [37] even if structural disease shrinkage has been observed only in a few cases. Employ of checkpoint blockade (e.g., pembrolizumab) has been explored with a weak activity (ORR 12\%) even if objective responses have been observed only in high-risk histotypes [38].

ReRT should be usually proposed as post-operative treatment, since it is related to prolonged survival rates, especially for ACC [39]: in our cohort, 5 patients out of $13(38 \%)$ received a post-operative RT [any energy]. Indications for post-operative RT include advanced stage (T3, T4, n+), positive surgical margins, high tumor grade, perineural invasion, recurrent diseases [40,41]. Moreover, reRT should be taken into account whenever surgery is not feasible, even though no data from prospective randomized data are available in the literature. Nowadays, state-of-the-art RT techniques, such as IMRT and stereotactic body RT (SBRT) allow steep dose gradients with optimal coverage even for complex target volumes, while allowing a good spare of close healthy tissues. Following NCCN recommendations, reRT should take place at least 6 months after the end of the first RT course and target volumes should be taken as narrower as possible, avoiding elective nodes coverage. Results are encouraging: Karam et al. re-treated 18 consecutive patients with SBRT with a median dose of 30 Gy delivered in 5 fractions. With a median cumulative dose of $91.1 \mathrm{~Gy}$, 2-years local control and OS were $53 \%$ and 39\%, respectively, even if 4 patients eventually experienced soft tissue necrosis as a late severe toxicity [42]. 
In regards to IMRT, most of the experience in literature refers to mixed series where the number of SGCs is significantly lower than pharyngeal or laryngeal squamous cell carcinoma cases $[19,43,44]$. Recently, Orlandi et al. analyzed reRT outcomes on a cohort of 159 patients, of which 71 (45\%) were not squamous cell carcinomas (in particular, 25 (16\%) were ACC and $8(5 \%)$ were affected by SGCs). With a median follow up of 49.9 months, 5-years OS and PFS were $43.5 \%$ (95\% CI, 34.6-54.8\%) and 20.9\% (95\% CI, 14.7-29.6\%), respectively. It is hard to make comparisons with our current results, due to the limited number of patients and the mixed cohort of the cited study. To our knowledge, there are no data available in literature concerning reRT on an exclusive ri-SGCs cohort.

Treatment with hadrontherapy represents a new approach considering that most of these tumors arose in a previously operated and irradiated field. PT and CIRT seem to be a reasonable choice to overcome dosimetric thresholds in unresectable patients. Two patients (one teenager) received PT and CIRT (delivered doses: PT 59.4 Gy(RBE) + CIRT 6 Gy(RBE); CIRT 68.8 Gy(RBE)). The physical properties of PT can be exploited in pediatric patients. Through PT nearly the same physical dose of XRT can be delivered, with a neat fall of dose outside the target: Grant et al. reported a $53 \%$ of grade $2^{-}$grade 3 (G2-G3) dermatitis, $0 \%$ of G2-G3 dysphagia, and a $46 \%$ of G2-G3 mucositis in a cohort of 13 pediatric patients receiving $60 \mathrm{~Gy}(\mathrm{RBE})$ ), compared to 11 patients treated with XRT (60 Gy, 54\%, $27 \%$ and $91 \%$ of toxicities rates, respectively). Takagi et al. compared PT and CIRT in a cohort of $80 \mathrm{pts}$ treated for head and neck ACC: no differences were found in terms of local control (66\% for PT, $68 \%$ for CIRT) for T4 unresectable cases when a dose of $65.0 \mathrm{~Gy}(\mathrm{RBE})$ was delivered. CIRT combines the ballistic properties and superior biological equivalent dose, allowing to retreat unresectable radio-resistant tumors. With a median CIRT dose of 51 Gy(RBE) $(27 \times 3$ Gy(RBE)) delivered 61 months after the first radiotherapy course, Jensen et al. reported an objective response rate of $57 \%$ and a local control (median follow-up 14 months) of 70\%; in that cohort, median cumulative dose was $128 \mathrm{~Gy}(\mathrm{RBE})$, with acceptable toxicity rates $[45,46]$. The Italian experience concerning reRT of SGCs with CIRT has been recently published [47]: from 2013 to 2016, 51 patients were treated with a median dose of $60 \mathrm{~Gy}(\mathrm{RBE})$ at 3-5 Gy(RBE) per fraction. After a median follow-up of 19 months, the local control rate was $41.2 \%$; one-year OS and PFS were $90.2 \%$ and $71.7 \%$, respectively.

In line with other papers, the treatment was well tolerated, with no G4 or G5 toxicities and only $17.5 \%$ of G3 events. Moreover, compared to other series with CIRT [46,48], reRT at CNAO did not suffer from soft tissue necrosis or carotid blow-out syndrome events [47]. This was probably related to the lower biologically equivalent doses (BED) delivered-155.2-167.4 Gy(RBE) — which could likely be increased in order to improve the outcomes. Interestingly, Vischioni et al. reported a shorter median LT, 6.33 years (range 1.08-20) compared to our results.

Despite the clinical challenges facing by any HNC specialist (i.e., radiation oncologist, head and neck surgeons, oro-maxillofacial surgeons, medical oncologist) Gs, a multidisciplinary approach is needed when dealing with ri-SC. This approach has been strongly recommended to achieve the best oncologic outcome and prevent or adequately treat any adverse effects [49-51]. Indeed, it should be considered as mandatory for SGCs as well [52]. In our series, most patients did not receive radical surgery; this is not an unexpected finding since post-treatment fibrosis and distortion of anatomy due to the treatment[s] for the previous tumor may increase the rate of positive margins. In this scenario, post-operative RT can almost double local control rate [53], and our results confirm that among 5 patients receiving marginal Sx and post-operative RT, 4 (80\%) had no evidence of disease at last follow up; the remaining one did, but he actually received CIRT. Data on the radiobiological response to this type of RT are still being collected, and a stable disease does not always translate into treatment failure [54]. Nevertheless, all of our patients treated by Sx and post-operative RT were alive at last follow up. In this context, a dedicated pathologist is advisable as SGCs show the greater discrepancy between the initial and definitive pathological diagnosis [55]; moreover, pathology must guide systemic therapy by identifying druggable targets. This approach allowed almost half of our patients to benefit from ADT, warranting at least 12 months of survival even without a curative treatment. Lastly, a multidisciplinary approach allows late toxicities to be more promptly identified 
and managed; this could explain the low number of severe sequelae among our patients and could result in a better quality of life.

RT is a well-known causative agent in the development of secondary solid tumors and the issue of second cancers following therapeutic radiation for a wide variety of malignancies is currently receiving increasing attention. Indeed, it is well recognized that patients receiving radiation therapy have a higher long-term risk for developing second primary cancers compared with patients who do not [13].

In pediatric patients, who constitute most of the cases originally treated in this study, germline mutations and hereditary conditions may place one at risk for developing a secondary malignancy, and may not be related solely to chemotherapy [25]. Considering that 10 patients in our series received radiation and chemotherapy compared to 3 patients who received radiation alone, our series suggests that the association of chemo-radiation may increase the risk of second SGCs compared to radiation alone. This may lead to the idea of treatment-induced second SGCs, rather than simply radio-induced secondary malignancies.

The main limitations of our research are its descriptive nature and the limited patient cohort. Beal KB et al. reported 18 radiation-induced salivary gland tumors, 15 of which were ri-SGCs [11]. The main strengths of this work are central pathologic review of the 12 non-ACC tumor specimens, the prolonged follow-up, and the details about treatments for ri-SGCs.

\section{Materials and Methods}

We retrospectively analyzed clinical data of consecutive patients with ri-SGCs referring to Fondazione IRCCS Istituto Nazionale dei Tumori, Milan, Italy, and to Centro Nazionale di Adroterapia Oncologica (CNAO, National Center of Oncological Hadrontherapy), Pavia, Italy, between 2015 and 2019. Patients were managed by head and neck and pediatric multidisciplinary teams established at both the former Institutions and involving dedicated radiation oncologists. We considered as eligible adult and pediatric patients with a confirmed pathological diagnosis of SGC, who received for previous tumors curative RT in $\mathrm{HN}$ areas, lung, and upper mediastinum, with or without concurrent or sequential chemotherapy. Multidisciplinary discussion has been considered within the inclusion criteria as well. Patients with primary leukemia, benign lesions, and patients who received only chemotherapy as a curative treatment have been excluded.

Staging procedures included $\mathrm{HN}$ computed tomography (CT) and/or magnetic resonance imaging (MRI), thorax and abdomen CT scan, or 18-FluoroDeoxyGlucose (FDG) Positron Emission Tomography (PET) total body scan.

Patients' characteristics, first tumor diagnosis, and secondary SGCs features, latent time (LT) from initial treatment to the development of SGCs, treatment, and outcome for secondary SGCs were analyzed.

Revision of all specimens was performed by a dedicated HN pathologist (PQ). Immunohistochemical (IHC) profile including androgen receptors and HER2 has been done in SDCs and adenocarcinoma not otherwise specified (NOS). Next-generation sequencing (NGS) has been performed on the tumor specimen until it was available for free (September 2019). RT plan of the primary tumor, when possible, was reconstructed on CT planning of the secondary SGCs with the aim of searching for a relationship between the dose distribution of the first RT and the second malignant tumor. Median overall survival (OS) and progression-free survival (PFS) and their 95\% confidence interval (CI) were estimated using the Kaplan-Meier method. Further subgroup analyses were realized according to histological features of SGCs.

This study was approved on June 2020 by the Ethical Committee of Fondazione IRCCS Istituto Nazionale dei Tumori, Milan, Italy (internal study identification number: INT 127-20). 


\section{Conclusions}

Our findings underline the benefit of a multidisciplinary approach and intraspeciality care for the management of this complex clinical scenario. This is realized through tailored treatments which are properly balanced between tumor control and toxicity, as to allow similar outcome to ex novo SGCs.

Author Contributions: Conceptualization, L.D.L. and E.O.; Data curation, D.A.R., Z.I., S.C., L.D.L. and E.O.; Formal analysis, S.C.; Funding acquisition, E.O.; Investigation, D.A.R., S.C., L.D.L. and E.O.; Methodology, S.C., L.D.L. and E.O.; Project administration, S.C., L.L., L.D.L. and E.O.; Resources, D.A.R., Z.I., S.C., B.V., L.G., A.I., N.A.I., L.L., M.G., C.P., D.L., B.D., P.Q., G.C., L.D.L. and E.O.; Supervision, D.A.R., Z.I., S.C., B.V., L.G., A.I., N.A.I., L.L., M.G., C.P., D.L., B.D., P.Q., G.C., L.D.L. and E.O.; Writing-original draft, D.A.R., Z.I., S.C., L.D.L. and E.O.; Writing-review \& editing, D.A.R., Z.I., S.C., B.V., L.G., A.I., N.A.I., L.L., M.G., C.P., D.L., B.D., P.Q., G.C., L.D.L. and E.O. All authors have read and agreed to the published version of the manuscript.

Funding: This research received no external funding.

Conflicts of Interest: Lisa Licitra declares the following conflicts of interests. Grants/research supports (Funds received by my institution for clinical studies and research activities in which I am involved): Astrazeneca, BMS, Boehringer Ingelheim, Celgene International, Debiopharm International SA, Eisai, Exelixis inc, Hoffmann-La Roche ltd, IRX Therapeutics inc, Medpace inc, Merck-Serono, MSD, Novartis, Pfizer, Roche. Honoraria or consultation fees [for public speaking/teaching in medical meetings and/or for expert opinion in advisory boards]: Astrazeneca, Bayer, BMS, Eisai, MSD, Merck-Serono, Boehringer Ingelheim, Novartis, Roche, Debiopharm International SA, Sobi, Ipsen, Incyte Biosciences Italy srl, Doxa Pharma, Amgen, Nanobiotics Sa and GSK. Public speaking/teaching from research companies \& commercial education providers: AccMed, Medical Science Fundation G. Lorenzini, Associazione Sinapsi, Think 2 IT, Aiom Servizi, Prime Oncology, WMA Congress Education, Fasi, DueCi promotion Srl, MI\&T, Net Congress \& Education, PRMA Consulting, Kura Oncology, Health \& Life srl, Immuno-Oncology Hub. Laura Deborah Locati reports grant and other financial relationship with Eisai, Ipsen, Merck Serono, MSD, BMS; Lilly. The remaining authors declare no conflict of interest.

\section{References}

1. RARECARENet. Available online: http://app.rarecarenet.eu (accessed on 12 December 2020).

2. Östman, J.; Anneroth, G.; Gustafsson, H.; Tavelin, B. Malignant salivary gland tumours in Sweden 1960-1989-An epidemiological study. Oral Oncol. 1997, 33, 169-176. [CrossRef]

3. Chiaravalli, S.; Guzzo, M.; Bisogno, G.; De Pasquale, M.D.; Migliorati, R.; De Leonardis, F.; Collini, P.; Casanova, M.; Cecchetto, G.; Ferrari, A. Salivary gland carcinomas in children and adolescents: The Italian TREP project experience: Salivary Gland Carcinoma in Children. Pediatr. Blood Cancer 2014, 61, 1961-1968. [CrossRef] [PubMed]

4. Saku, T.; Hayashi, Y.; Takahara, O.; Matsuura, H.; Tokunaga, M.; Tokuoka, S.; Soda, M.; Mabuchi, K.; Land, C.E. Salivary gland tumors among atomic bomb survivors, 1950-1987. Cancer 1997, 79, 1465-1475. [CrossRef]

5. Land, C.E.; Saku, T.; Hayashi, Y.; Takahara, O.; Matsuura, H.; Tokuoka, S.; Tokunaga, M.; Mabuchi, K. Incidence of salivary gland tumors among atomic bomb survivors, 1950-1987. Evaluation of Radiation-Related Risk. Radiat. Res. 1996, 146, 28-36. [CrossRef]

6. Modan, B.; Chetrit, A.; Alfandary, E.; Tamir, A.; Lusky, A.; Wolf, M.; Shpilberg, O. Increased risk of salivary gland tumors after low-dose irradiation. Laryngoscope 1998, 108, 1095-1097. [CrossRef]

7. Schneider, A.B.; Lubin, J.; Ron, E.; Abrahams, C.; Stovall, M.; Goel, A.; Shore-Freedman, E.; Gierlowski, T.C. Salivary gland tumors after childhood radiation treatment for benign conditions of the head and neck: Dose-response relationships. Radiat. Res. 1998, 149, 625-630. [CrossRef]

8. Bassal, M.; Mertens, A.C.; Taylor, L.; Neglia, J.P.; Greffe, B.S.; Hammond, S.; Ronckers, C.M.; Friedman, D.L.; Stovall, M.; Yasui, Y.Y.; et al. Risk of selected subsequent carcinomas in survivors of childhood cancer: A report from the childhood cancer survivor study. J. Clin. Oncol. 2006, 24, 476-483. [CrossRef]

9. Verma, J.; The, B.S.; Paulino, A.C. Characteristics and outcome of radiation and chemotherapy-related mucoepidermoid carcinoma of the salivary glands. Pediatr. Blood Cancer 2011, 57, 1137-1141. [CrossRef]

10. Zidar, N.; Gale, N.; Župevc, A.; Žargi, M. Salivary gland tumours as second neoplasms: Two cases and literature review. J. Laryngol. Otol. 2010, 124, 577-580. [CrossRef]

11. Beal, K.P.; Singh, B.; Kraus, D.; Yahalom, J.; Portlock, C.; Wolden, S.L. Radiation-induced salivary gland tumors: A report of 18 cases and a review of the literature. Cancer J. 2003, 9, 467-471. [CrossRef] 
12. Védrine, P.O.; Coffinet, L.; Temam, S.; Montagne, K.; Lapeyre, M.; Oberlin, O.; Orbach, D.; Simon, C.; Sommelet, D. Mucoepidermoid carcinoma of salivary glands in the pediatric age group: 18 clinical cases, including $11 \mathrm{~s}$ malignant neoplasms. Head Neck 2006, 28, 827-833. [CrossRef] [PubMed]

13. Whatley, W.; Thompson, J.; Rao, B. Salivary gland tumors in survivors of childhood cancer. Otolaryngol. Head Neck Surg. 2006, 134, 385-388. [CrossRef] [PubMed]

14. Orlandi, E.; Iacovelli, N.A.; Bonora, M.; Cavallo, A.; Fossati, P. Salivary gland. Photon beam and particle radiotherapy: Present and future. Oral Oncol. 2016, 60, 146-156. [CrossRef] [PubMed]

15. Adelstein, D.J.; Koyfman, S.A.; El-Naggar, A.K.; Hanna, E.Y. Biology and management of salivary gland cancers. Semin. Radiat. Oncol. 2012, 22, 245-253. [CrossRef]

16. Paterson, N.J.; Ramadan, O. Radiation-induced parotid mucoepidermoid carcinoma: A systematic review. Otolaryngol. Open J. 2017, 3, 9-15.

17. Armstrong, G.T.; Liu, W.; Leisenring, W.; Yasui, Y.; Hammond, S.; Bhatia, S.; Neglia, J.P.; Stovall, M.; Srivastava, D.; Robison, L.L. Occurrence of multiple subsequent neoplasms in long-term survivors of childhood cancer: A report from the childhood cancer survivor study. J. Clin. Oncol. 2011, 29, 3056-3064. [CrossRef]

18. Bhattacharyya, N.; Fried, M.P. Determinants of survival in parotid gland carcinoma: A population-based study. Am. J. Otolaryngol. 2005, 26, 39-44. [CrossRef]

19. Orlandi, E.; Bonomo, P.; Ferella, L.; D’Angelo, E.; Maddalo, M.; Alterio, D.; Infante, G.; Bacigalupo, A.; Argenone, A.; Iacovelli, N.A.; et al. Long-term outcome of re-irradiation for recurrent or second primary head and neck cancer: A multi-institutional study of AIRO-Head and Neck working group. Head Neck 2019, 41, 3684-3692. [CrossRef]

20. Chowdhry, A.K.; McHugh, C.; Fung, C.; Dhakal, S.; Constine, L.S.; Milano, M.T. Second primary head and neck cancer after Hodgkin lymphoma: A population-based study of 44,879 survivors of Hodgkin lymphoma: Head and Neck Cancer After Hodgkin Lymphoma. Cancer 2015, 121, 1436-1445. [CrossRef]

21. Boukheris, H.; Stovall, M.; Gilbert, E.S.; Stratton, K.L.; Smith, S.A.; Weathers, R.; Hammond, S.; Mertens, A.C.; Donaldson, S.S.; Armstrong, G.T.; et al. Risk of Salivary Gland Cancer Following Childhood Cancer: A Report From The Childhood Cancer Survivor Study. Int. J. Radiat. Oncol. 2013, 85, 776-783. [CrossRef]

22. Bjordal, K.; Ahlner-Elmqvist, M.; Hammerlid, E.; Boysen, M.; Evensen, J.F.; Biorklund, A.; Jannert, M.; Westin, T.; Kaasa, S. A prospective study of quality of life in head and neck cancer patients. Part II: Longitudinal data. Laryngoscope 2001, 111, 1440-1452. [CrossRef] [PubMed]

23. Atula, T.; Grénman, R.; Klemi, P.; Syrjänen, S. Human papillomavirus, Epstein-Barr virus, human herpesvirus 8 and human cytomegalovirus involvement in salivary gland tumours. Oral Oncol. 1998, 34, 391-395. [CrossRef]

24. Boukheris, H.; Ron, E.; Dores, G.M.; Stovall, M.; Smith, S.A.; Curtis, R.E. Risk of radiation-related salivary gland carcinomas among survivors of Hodgkin lymphoma: A population-based analysis. Cancer 2008, 113, 3153-3159. [CrossRef] [PubMed]

25. Rutigliano, D.N.; Meyers, P.; Ghossein, R.A.; Carlson, D.L.; Kayton, M.L.; Kraus, D.; La Quaglia, M.P. Mucoepidermoid carcinoma as a secondary malignancy in pediatric sarcoma. J. Pediatr. Surg. 2007, 42, 9-13. [CrossRef] [PubMed]

26. Locati, L.D.; Perrone, F.; Cortelazzi, B.; Lo Vullo, S.; Bossi, P.; Dagrada, G.; Quattrone, P.; Bergamini, C.; Potepan, P.; Civelli, E.; et al. Clinical activity of androgen deprivation therapy in patients with metastatic/relapsed androgen receptor-positive salivary gland cancers: Androgen deprivation therapy in salivary gland cancers. Head Neck 2016, 38, 724-731. [CrossRef] [PubMed]

27. Fushimi, C.; Tada, Y.; Takahashi, H.; Nagao, T.; Ojiri, H.; Masubuchi, T.; Matsuki, T.; Miura, K.; Kawakita, D.; Hirai, H.; et al. A prospective phase II study of combined androgen blockade in patients with androgen receptor-positive metastatic or locally advanced unresectable salivary gland carcinoma. Ann. Oncol. 2018, 29, 979-984. [CrossRef] [PubMed]

28. Alfieri, S.; Granata, R.; Bergamini, C.; Resteghini, C.; Bossi, P.; Licitra, L.F.; Locati, L.D. Systemic therapy in metastatic salivary gland carcinomas: A pathology-driven paradigm? Oral Oncol. 2017, 66, 58-63. [CrossRef]

29. Locati, L.D.; Perrone, F.; Losa, M.; Mela, M.; Casieri, P.; Orsenigo, M.; Cortelazzi, B.; Negri, T.; Tamborini, E.; Quattrone, P.; et al. Treatment relevant target immunophenotyping of 139 salivary gland carcinomas (SGCs). Oral Oncol. 2009, 45, 986-990. [CrossRef]

30. Skálová, A.; Vanecek, T.; Sima, R.; Laco, J.; Weinreb, I.; Perez-Ordonez, B.; Starek, I.; Geierova, M.; Simpson, R.H.; Passador-Santos, F.; et al. Mammary analogue secretory carcinoma of salivary glands, 
containing the ETV6-NTRK3 fusion gene: A hitherto undescribed salivary gland tumor entity. Am. J. Surg. Pathol. 2010, 34, 599-608. [CrossRef]

31. Skálová, A.; Vanecek, T.; Uro-Coste, E.; Bishop, J.A.; Weinreb, I.; Thompson, L.D.R.; de Sanctis, S.; Schiavo-Lena, M.; Laco, J.; Badoual, C.; et al. Molecular profiling of salivary gland intraductal carcinoma revealed a subset of tumors harboring NCOA4-RET and novel TRIM27-RET fusions: A report of 17 cases. Am. J. Surg. Pathol. 2018, 42, 1445-1455. [CrossRef]

32. Nakaguro, M.; Urano, M.; Ogawa, I.; Hirai, H.; Yamamoto, Y.; Yamaguchi, H.; Tanigawa, M.; Matsubayashi, J.; Hirano, H.; Shibahara, J.; et al. Histopathological evaluation of minor salivary gland papillary-cystic tumours: Focus on genetic alterations in sialadenoma papilliferum and intraductal papillary mucinous neoplasm. Histopathology 2020, 76, 411-422. [CrossRef] [PubMed]

33. Corrêa, T.S.; Matos, G.D.R.; Segura, M.; dos Anjos, C.H. Second-line treatment of HER2-positive salivary gland tumor: Ado-trastuzumab emtansine (T-DM1) after progression on trastuzumab. Case Rep. Oncol. 2018, 11, 252-257. [CrossRef] [PubMed]

34. Drilon, A.; Li, G.; Dogan, S.; Gounder, M.; Shen, R.; Arcila, M.; Wang, L.; Hyman, D.M.; Hechtman, J.; Wei, G.; et al. What hides behind the MASC: Clinical response and acquired resistance to entrectinib after ETV6-NTRK3 identification in a mammary analogue secretory carcinoma (MASC). Ann. Oncol. 2016, 27, 920-926. [CrossRef] [PubMed]

35. Drilon, A.; Laetsch, T.W.; Kummar, S.; DuBois, S.G.; Lassen, U.N.; Demetri, G.D.; Nathenson, M.; Doebele, R.C.; Farago, A.F.; Pappo, A.S.; et al. Efficacy of Larotrectinib in TRK Fusion-Positive Cancers in Adults and Children. N. Engl. J. Med. 2018, 378, 731-739. [CrossRef]

36. Moore, A.; Bar, Y.; Maurice-Dror, C.; Ospovat, I.; Sarfaty, M.; Korzets, Y.; Goldvaser, H.; Gordon, N.; Billan, S.; Gutfeld, O.; et al. Next-generation sequencing in salivary gland carcinoma: Targetable alterations lead to a therapeutic advantage-Multicenter experience. Head Neck 2020, 42, 599-607. [CrossRef]

37. Locati, L.D.; Galbiati, D.; Calareso, G.; Alfieri, S.; Singer, S.; Cavalieri, S.; Bergamini, C.; Bossi, P.; Orlandi, E.; Resteghini, C.; et al. Patients with adenoid cystic carcinomas of the salivary glands treated with lenvatinib: Activity and quality of life. Cancer 2020, 126, 1888-1894. [CrossRef]

38. Cohen, R.B.; Delord, J.-P.; Doi, T.; Piha-Paul, S.A.; Liu, S.V.; Gilbert, J.; Algazi, A.P.; Damian, S.; Hong, R.L.; Le Tourneau, C.; et al. Pembrolizumab for the treatment of advanced salivary gland carcinoma: Findings of the phase 1b KEYNOTE-028 study. Am. J. Clin. Oncol. 2018, 41, 1083. [CrossRef]

39. Simpson, J.R.; Thawley, S.E.; Matsuba, H.M. Adenoid cystic salivary gland carcinoma: Treatment with irradiation and surgery. Radiology 1984, 151, 509-512. [CrossRef]

40. Ali, S.; Palmer, F.L.; Yu, C.; DiLorenzo, M.; Shah, J.P.; Kattan, M.W.; Patel, S.G.; Ganly, I. A predictive nomogram for recurrence of carcinoma of the major salivary glands. JAMA Otolaryngol. Neck Surg. 2013, 139, 698-705.

41. Ali, S.; Palmer, F.L.; Yu, C.; DiLorenzo, M.; Shah, J.P.; Kattan, M.W.; Patel, S.G.; Ganly, I. Postoperative nomograms predictive of survival after surgical management of malignant tumors of the major salivary glands. Ann. Surg. Oncol. 2014, 21, 637-642. [CrossRef]

42. Karam, S.D.; Snider, J.W.; Wang, H.; Wooster, M.; Lominska, C.; Deeken, J.; Newkirk, K.; Davidson, B.; Harter, K.W. Reirradiation of recurrent salivary gland malignancies with fractionated stereotactic body radiation therapy. J. Radiat. Oncol. 2012, 1, 147-153. [CrossRef]

43. Zwicker, F.; Roeder, F.; Hauswald, H.; Thieke, C.; Timke, C.; Schlegel, W.; Debus, J.; Münter, M.W.; Huber, P.E. Reirradiation with intensity-modulated radiotherapy in recurrent head and neck cancer. Head Neck 2011, 33, 1695-1702. [CrossRef] [PubMed]

44. Ward, M.C.; Riaz, N.; Caudell, J.J.; Dunlap, N.E.; Isrow, D.; Zakem, S.J.; Dault, J.; Awan, M.J.; Vargo, J.A.; Heron, D.E.; et al. Refining patient selection for reirradiation of head and neck squamous carcinoma in the IMRT era: A multi-institution cohort study by the MIRI collaborative. Int. J. Radiat. Oncol. Biol. Phys. 2018, 100, 586-594. [CrossRef] [PubMed]

45. Jensen, A.D.; Nikoghosyan, A.; Ellerbrock, M.; Ecker, S.; Debus, J.; Münter, M.W. Re-irradiation with scanned charged particle beams in recurrent tumours of the head and neck: Acute toxicity and feasibility. Radiother. Oncol. J. Eur. Soc. Ther. Radiol. Oncol. 2011, 101, 383-387. [CrossRef] [PubMed] 
46. Jensen, A.D.; Poulakis, M.; Nikoghosyan, A.V.; Chaudhri, N.; Uhl, M.; Münter, M.W.; Jensen, A.D.; Poulakis, M.; Nikoghosyan, A.V.; Chaudhri, N.; et al. Re-irradiation of adenoid cystic carcinoma: Analysis and evaluation of outcome in 52 consecutive patients treated with raster-scanned carbon ion therapy. Radiother. Oncol. J. Eur. Soc. Ther. Radiol. Oncol. 2015, 114, 182-188. [CrossRef] [PubMed]

47. Vischioni, B.; Dhanireddy, B.; Severo, C.; Bonora, M.; Ronchi, S.; Vitolo, V.; Fiore, M.R.; D’Ippolito, E.; Petrucci, R.; Barcellini, A.; et al. Reirradiation of salivary gland tumors with carbon ion radiotherapy at CNAO. Radiother. Oncol. 2020, 145, 172-177. [CrossRef]

48. Hayashi, K.; Hayashi, K.; Koto, M.; Ikawa, H.; Hagiwara, Y.; Tsuji, H.; Ogawa, K.; Kamada, T. Feasibility of Re-irradiation using carbon ions for recurrent head and neck malignancies after carbon-ion radiotherapy. Radiother. Oncol. 2019, 136, 148-153. [CrossRef] [PubMed]

49. Orlandi, E.; Alfieri, S.; Simon, C.; Trama, A.; Licitra, L.; RARECAREnet Working Group. Treatment challenges in and outside a network setting: Head and neck cancers. Eur. J. Surg. Oncol. 2019, 45, 40-45. [CrossRef]

50. Pfister, D.G.; Spencer, S.; Adelstein, D.; Adkins, D.; Anzai, Y.; Brizel, D.M.; Bruce, J.Y.; Busse, P.M.; Caudell, J.J.; Cmelak, A.J.; et al. Head and neck cancers, version 2.2020, NCCN clinical practice guidelines in oncology. J. Natl. Compr. Canc. Netw. 2020, 18, 873-898. [CrossRef]

51. Jensen, K.; Friborg, J.; Hansen, C.R.; Samsøe, E.; Johansen, J.; Andersen, M.; Smulders, B.; Andersen, E.; Nielsen, M.S.; Eriksen, J.G.; et al. The Danish Head and Neck Cancer Group (DAHANCA) 2020 radiotherapy guidelines. Radiother. Oncol. 2020, 151, 149-151. [CrossRef]

52. Sood, S.; McGurk, M.; Vaz, F. Management of salivary gland tumours: United Kingdom national multidisciplinary guidelines. J. Laryngol. Otol. 2016, 130, S142-S149. [CrossRef] [PubMed]

53. Terhaard, C.H.J.; Lubsen, H.; Rasch, C.R.N.; Levendag, P.C.; Kaanders, H.H.À.M.; Tjho-Heslinga, R.E.; van Den Ende, P.L.; Burlage, F.; Dutch Head and Neck Oncology Cooperative Group. The role of radiotherapy in the treatment of malignant salivary gland tumors. Int. J. Radiat. Oncol. 2005, 61, 103-111. [CrossRef] [PubMed]

54. Hayashi, K.; Yamamoto, N.; Shirai, T.; Takeuchi, A.; Kimura, H.; Miwa, S.; Higuchi, T.; Abe, K.; Taniguchi, Y.; Aiba, H.; et al. Sequential histological findings and clinical response after carbon ion radiotherapy for unresectable sarcoma. Clin. Transl. Radiat. Oncol. 2017, 2, 41-45. [CrossRef] [PubMed]

55. Bergamini, C.; Locati, L.; Bossi, P.; Granata, R.; Alfieri, S.; Resteghini, C.; Imbimbo, M.; Fallai, C.; Orlandi, E.; Tana, S.; et al. Does a multidisciplinary team approach in a tertiary referral centre impact on the initial management of head and neck cancer? Oral Oncol. 2016, 54, 54-57. [CrossRef] [PubMed]

Publisher's Note: MDPI stays neutral with regard to jurisdictional claims in published maps and institutional affiliations.

(C) 2020 by the authors. Licensee MDPI, Basel, Switzerland. This article is an open access article distributed under the terms and conditions of the Creative Commons Attribution (CC BY) license (http://creativecommons.org/licenses/by/4.0/). 